\title{
Population genomics for fruitflies
}

Starve a fruitfly for a couple of hours, and it gets a little cranky. Pop it into a fruit-fly-sized ring, add seven other starving flies and just one piece of food, and you'll have a riot. "They stand up on their little hind legs and tussle," says geneticist Trudy Mackay at North Carolina State University in Raleigh. "And then there's the wing slap," adds her collaborator, Robert Anholt.

Researchers in Mackay's lab quantify aggressive behaviour by counting how often each fruitfly wrestles, slaps, or chases its competitors. They have uncovered a wide range of responses, even among members of the same species. One fly had 100 aggressive interactions in two minutes; others had as few as three. "Those are the pacifists," says Mackay. "They sit there and share the food." Anholt sums up the data succinctly: "There's enormous variation - from flies that are real wimps to flies that really beat the shit out of each other."

It's that sort of variation that has inspired Mackay and her colleagues to propose an ambitious new drosophila genomics project: sequencing the genomes of 192 flies. The aim is to use this information to understand the genetic changes underlying the variation in behaviour and appearance in natural populations. By breaking free from lab strains that have spent decades living in vials on lumps of smelly, sterilized lab food, researchers hope to get a better glimpse of the evolutionary forces at work in fly populations. "The history of a population can leave a kind of footprint on the genome," says fruit-fly researcher Esteban Hasson of the University of Buenos Aires. "Perhaps we will be able to map the mechanisms that shaped the variation found in these flies."

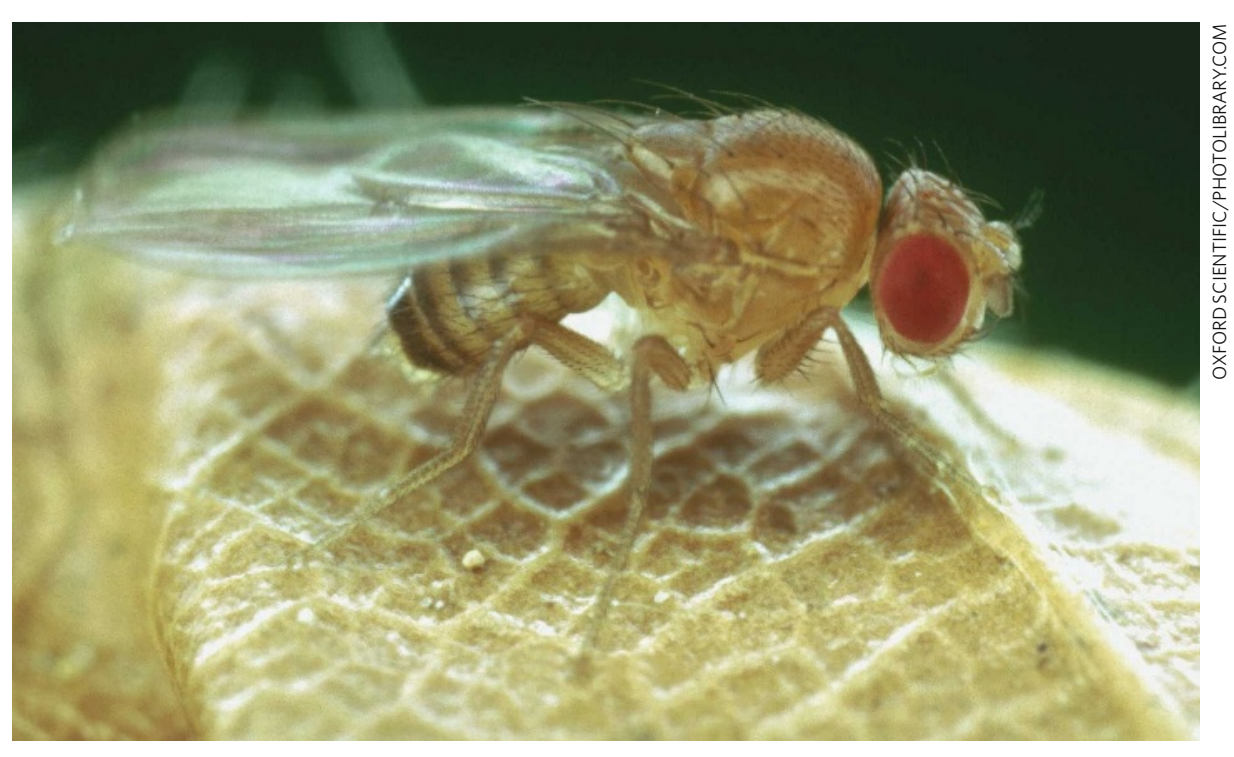

Researchers want to sequence the genomes of a swarm of the geneticist's favourite fly.

The flies are all members of the same species - the classic genetic model organism Drosophila melanogaster — but, unlike some lab populations, they have been kept in the lab for only five years - since their ancestors were trapped at a farmers' market in Raleigh. They have been inbred for 20 generations in the lab to produce a collection of 'pure-bred' flies that are amenable to genetic analysis. Flies obtained from the wild have been used to study natural variation before, but the work can be arduous. Having full genome sequences promises to dramatically speed up the process, says Mackay.

This project, which was recently approved by the US National Human Genome Research

\section{What's the magic number?}

As the price of large sequencing projects drops, researchers are launching ever more ambitious plans to 'resequence' full genomes. These projects aim to sequence additional individuals from a species that has already had its genome sequenced. For example, it's more than a decade since the genome of the model yeast Saccharomyces cerevisiae was published, and since then 36 strains isolated from the wild have been fully sequenced.

But the multicellular model organisms, with their bulky genomes, have not kept pace. Fifteen strains of mice have been resequenced and three wild isolates of the nematode Caenorhabditis elegans, with plans to sequence a dozen more. And the full genome sequences of just two people have been published, geneticists Craig Venter and James Watson.

The numbers are set to go much higher. The most famous resequencing endeavour is the 1000 Genomes Project, which aims to sequence the genomes of 1,000 people. Less well known is another project, still in the planning stage, that hopes to literally one-up the 1000 Genomes Project: the proposed 1001 Genomes Project, headed by Detlef Weigel at the University of Tübingen, Germany, to sequence 1,001 different strains of the flowering plant Arabidopsis thaliana. In the meantime, Weigel has received funding to sequence 80 strains from around the world.
Institute in Bethesda, Maryland, is expected to cost around US\$4 million and to take about two years. Although similar projects are under way in other model organisms (see 'What's the magic number?'), most of those are smaller and will sequence only targeted regions of the genome. Even when a full genome is the goal, it is typically only a 'draft' sequence that contains a relatively high frequency of gaps and errors.

The drosophila project, in contrast, aims to produce high-quality sequence using two highthroughput sequencing methods with complementary strengths. The machines produced by Illumina of San Diego, California, are particularly good at detecting single base differences in the DNA sequence, whereas the sequencers from 454 Life Sciences of Branford, Connecticut, are better at finding regions in which large stretches of DNA have been inserted or deleted. "I'm after as much sequence as I can get, in a very greedy way," says Stephen Richards of Baylor College of Medicine in Houston, Texas, who is an investigator on the drosophila project.

This approach will provide a useful testing ground, says population geneticist Philip Awadalla of the University of Montreal in Canada. Awadalla usually studies genetics in humans and in the malaria parasite, but says the analytical tools developed in the drosophila project could be useful for his work. Awadalla also plans to analyse the drosophila sequences once they become available. The small genome and the ease with which different strains of D. melanogaster can be characterized provide clear advantages over humans or other insects, he says. 
Drosophila researchers are renowned for devising creative ways of studying their beloved flies. Researchers are already lining up to study Mackay's wild isolates, with plans to study learning and memory, wing morphology, body size, social behaviour, circadian rhythm and responses to different odours and drugs. Hasson will try to determine why some flies like to lay their eggs in grapes whereas others prefer oranges, and Anholt will test responses to alcohol using his 'inebriometer', a device that measures how quickly flies become woozy from ethanol fumes.

Richards says that similar projects in other insects, such as mosquitoes or honeybees, could be on the horizon. "The cost of sequencing is coming down so quickly; in the future it'll just be a normal grant proposal to do 500 insects," he says.

Heidi Ledford

ON THE RECORD “We have ICEI!!!! Yes, ICE, ${ }^{\star}$ WATER ICE` on Mars! w00t!!! Best day ever!!."

Comment from 19 June on the Mars Phoenix 'twitter' feed, where team members leave updates in the persona of their plucky lander.

\section{SCORECARD}

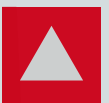

Runner reaction time Canadian scientists have found that being

close to the starting gun startles runners into a speedier start. The team suggests that Canada's Olympic runners wear cranked-up hearing aids in Beijing to get the best reaction time off the blocks.

As Europe's museums begin archiving their collections in digital format, skeletons are emerging - and not just of the physical variety. One South African tribe already says it will oppose the inclusion of images of its people's remains in any multimedia format.

The University of Vienna has started to digitize the collection made in the early twentieth century by Rudolf Pöch, considered one of anthropology's founding fathers. The project, headed by Maria Teschler-Nicola, will improve the collection's accessibility for researchers and store the delicate material in a sustainable way, using electronic records of physiological measurements as well as twoand three-dimensional scans.

But the full collection, which includes human remains and thousands of ethnographic artefacts, was gathered using unethical methods, such as grave-robbing.

Around the turn of the twentieth century, anthropological adventurers in search of exotic artefacts collected skeletal remains from ethnic groups in Africa, Asia and Australia, and sold them to museums in the West. There, they were often displayed in exhibitions purporting to show the evolution of humans from these supposedly 'primitive' origins.

Museums in Europe and the United States have now stopped displaying the remains of modern humans that were not acquired by donation. But it was not until 1995 that the Natural History Museum Vienna removed an exhibit depicting a Negro man as being below Caucasians on the evolutionary scale of development.

"There are maybe 300 sensitive cases in our collection," says Teschler-Nicola. "We don't want to repeat the same mistakes, but we don't have any guidelines." Such bones can be important research material for archaeoanthropologists, which complicates the museum's decision.

The Natural History Museum in London is also planning to digitally record its entire collection, and has yet to decide what to do about its own contentious human remains. The issue was raised at a meeting organized at the museum in March to survey the opinion of leading international scientists. An internal report from the meeting is thought to endorse continuing scientific study, including digitization, on human remains that may be subject to repatriation. "The decision on how to move forward is yet to be taken," says John Jackson, science-policy coordinator at the museum. "There are constraints on whether those remains should be in the collection - whether it is ethically right has to be considered very carefully."

"When repatriation requests are made there is an expectation that all studies have been done. This is not the case," says Robert Hedges, an archaeologist at the University of Oxford, UK, who attended the meeting. "There is every reason for studying remains that are vulnerable to repatriation. You have to be aware that one is liable to lose information if remains are repatriated."

Roger Chennells, legal adviser to the San Institute, a South African nongovernmental organization that campaigns for the repatriation of the aboriginal San people's remains, some of which are in the Pöch collection, told Nature. "We have not been consulted, and we do not support any photographic archiving of our people's remains - we are opposed to it," he says.

The University of Vienna and the National History Museum in London both hope to draw up guidelines in the next few weeks. Tony Scully

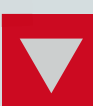

Mail delivery time A 'slow art' project at Bournemouth University in the United Kingdom uses three snails crawling around a tank to pick up e-mail signals and pass them on. The 'real snail mail' can take months to be delivered.

NUMBER CRUNCH 19,999.99 The 'N-prize' cash award for launching "an impossibly small satellite on a ludicrously small budget".

\&999.99 The maximum allowed cost of the launch.

9 orbits How far it has to fly.

9.99-19.99 grams

The required weight range of the satellite.

0.1-1 kilogram The weight range of the smallest common category of satellites, known as 'picosatellites'. One popular one, CubeSat, costs about $£ 20,000$ (US\$40,000) per launch.

The prize rules state that "imaginative use of string and chewing gum is encouraged".

Sources: Phoenix Twitter, Edmonton Journal, BBC, www.n-prize.com 